\title{
Improving stability and utilization of the electricity infrastructure of a neighborhood
}

\author{
Albert Molderink, Vincent Bakker, Maurice G.C. Bosman, Johann L. Hurink, Gerard J.M. Smit \\ University of Twente, Department of Computer Science, Mathematics and Electrical Engineering \\ P.O. Box 217, 7500 AE, Enschede, The Netherlands \\ email: a.molderink@utwente.nl
}

\begin{abstract}
Increasing energy prices and the greenhouse effect lead to more awareness of energy efficiency of electricity supply. During the last years, a lot of technologies and optimization methodologies are developed to increase the efficiency, maintain the grid stability and support large scale introduction of renewable sources. In previous work, we showed the effectiveness of our three-step methodology to reach these objective, consisting of 1) off-line prediction, 2) off-line planning and 3) online scheduling [1].

The goal of this paper is 1) to analyze the impact of installing a local controller in the house and 2) to analyze the stabilizing effect of the optimization algorithms on a large group of houses. To investigate whether it is possible to develop a local controller, a proof-of-concept is built using an embedded PC. The prototype consumes significantly less power than it can save. The stabilizing effect is studied by two large scale use cases. The first one is a simulation of 200 houses that together try to respond on fluctuation in generation of a windmill park. The second one is a simulation of a fleet of $\mathbf{1 0 0}$ electrical cars that need to be charged at night. Using the three step methodology in these two scenarios, the required balancing power, peaks and fluctuations in the required generation of the power plants decrease up to $40 \%$.
\end{abstract}

\section{INTRODUCTION}

In the last decades, more and more stress is put on the electricity supply and infrastructure. On the one hand, electricity usage increased significantly and became very fluctuating. Since the maximum peak usage defines the generation and grid capacity, the required capacity has increased. Furthermore, due to the fluctuations in usage (and therefore in required generation) the generation efficiency decreased [2].

On the other hand, reduction in the $\mathrm{CO}_{2}$ emissions and introduction of generation based on renewable sources are important topics today. However, these renewable resources are mainly given by very fluctuating and uncontrollable sun, water- and wind-power. The generation patterns resulting from these renewable sources may have some similarities with the electricity demand patterns, but they are not equal. For this reason, supplemental production is required to keep the demand and supply in balance, resulting in an even more fluctuating generation pattern for the conventional power plants. Finally, the introduction of new, energy efficient technologies such as electrical cars can result in an even further fluctuating electricity demand. If electrical cars are charged in an uncontrolled way, this may result in high peak demands of electricity since these vehicles often will be charged in the evening and need to be charged fast to ensure enough capacity for the upcoming trip. Lowering the peaks in demand is desirable to improve the usage of the available grid capacity and maintain a stable and reliable grid.

A solution for these problems may be to transform domestic customers from static consumers into active players in the production process. More and more new technologies with controllable load and generation are developed, such as controllable white goods and micro-generation. Furthermore, domestic energy storage of both heat and electricity is becoming quite common. Managing these new technologies has a optimization potential, since by managing these technologies the consumption pattern of houses can be partially controlled. The goal of our research is to determine a methodology to use this optimization potential to 1) optimize efficiency of current power plants, 2) support the introduction of a large penetration level of renewable sources (and thereby facilitate the means that are needed for $\mathrm{CO}_{2}$ reduction) and 3) optimize usage of the current grid capacity.

In [1] a control strategy is presented to exploit this optimization potential in a generic way. The methodology is flexible in both the optimization objective and the technologies available within houses. After all, objectives may differ over time and different houses may have different technologies installed. This control strategy is explained in more detail in Section II.

The first goal of this paper is to investigate whether it is possible to develop a local controller. Therefore, a proof-ofconcept is built using an embedded PC. This local controller should predict the energy production/consumption potential, optionally communicate with a global controller, monitor and manage domestic devices and run an optimization algorithm. It is based on the prototype described in [3], where a desktop PC is used. The prototype should consume less power than it can save.

The second goal is to verify the effect of our optimization methodology when it is applied on a large number of houses. This is done using a simulator [4]. Small scale scenarios are simulated and verified in previous work [1], [3] and one large scale simulation is performed [5]. However, the goal of this large scale simulation was to investigate whether it is possible to apply the methodology on a large group of houses and what the communication requirements are. No extensive verification of the optimization results are done.

The rest of this paper is built up as follows. The next section describes the developed optimization methodology with the underlying model and introduces the test cases. Section III describes the implemented local controller and the fourth section describes the simulations and simulation results of the 
test cases. In Section V we end up with a discussion.

\section{APPROACH}

In this section the optimization methodology and the underlying model are explained. Furthermore, the simulated use cases are introduced. The developed methodology to exploit the domestic optimization potential consists of three steps. These steps are explained in the following paragraphs, a detailed description can be found in [6].

In the first step, a system located at the consumers (local controller) predicts the production and consumption pattern for all appliances for the upcoming day. For example, in a normal household multiple appliances like a TV, washing machine, central heating are present. For each appliance, based on the historical usage pattern of the residents and external factors like the weather, a predicted energy profile is generated. Based on the expected energy profile and the characteristics of the devices the scheduling freedom and optimization potentials are determined. These potentials are aggregated by the local controller and sent to a global controller. The global controller is structured as a hierarchical tree for scalability and to reduce communication. In each node of the tree the received profiles are aggregated and sent upwards in the tree until the root node.

In the second step, these optimization potentials can be used by a central planner to exploit the potential to reach a global objective. The root node determines steering signals based on the received information and the objective. These steering signals are distributed via the tree structure, whereby each node may adjust the steering signals. Adjusted profiles are determined in the houses, based on the (new) steering signals and the predictions. These new profiles are again sent upwards. In this iterative way a near-optimal solution can be found with a reasonable computational time. Example objectives are peak shaving or compensating the fluctuation of the production of renewable sources like wind-parks. The result of the second step is a plan for each household for the upcoming day and an overall production/consumption profile.

In the final step a realtime control algorithm decides at which times appliances are switched on/off, when and how much energy flows from or to the buffers and when and which generators are switched on. This realtime control algorithm uses the steering signals from the global plan as input, but preserves the comfort of the residents in conflict situations. The steering signals from the global controller can be based on the planning as described before, but can also be based on a realtime algorithm on a global level. In the latter case, the local controllers send status information to the global controller. The global controller determines steering signals based on the status information and optionally on predictions of the consumption/production. The local controller can also run autonomously, for example when the connection with the global controller is lost.

The global controller uses predictions of the consumption/production patterns. For the iterative planning algorithm, predictions of individual patterns is required. For the realtime global control algorithm on total pattern might be sufficient. The actual consumption/production patterns often deviate from the predicted patterns, the optimization algorithms should be

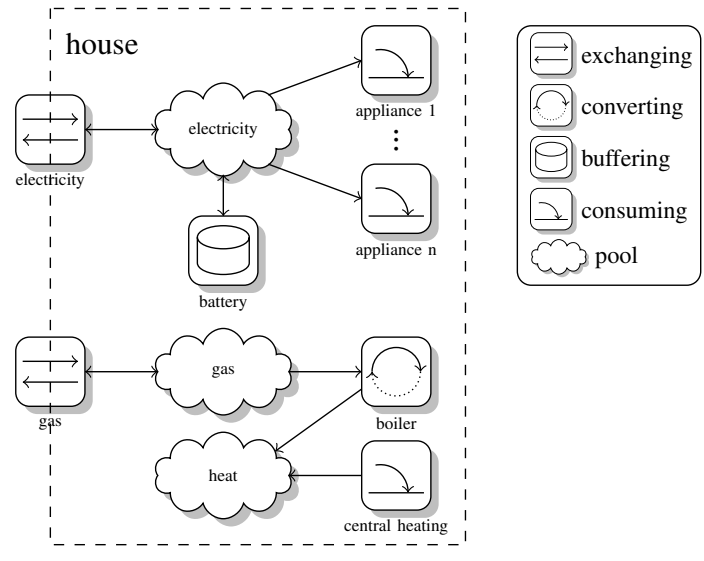

Fig. 1. Model of the house

capable of dealing with these prediction errors. Short term predictions are often more accurate than long term predictions, as well as predictions of the total pattern are often more accurate than predictions of individual patterns. However, the short term patterns and the pattern of the total consumption/production contain less information and less detailed plans can be made.

To analyze the energy-streams and optimization potential, a general model of the energy situation in a domestic environment has been set up. The basis of this model is a house with appliances. Since the behavior of individual devices is optimized, the detail level of the model is on a device level. Houses contain multiple devices and exchange energy with the environment (e.g. gas import, electricity import/export). Multiple houses can be combined in a grid to analyze their overall behavior. Based on this model, a simulator is built to be able to quickly simulate different scenarios, house configurations and device parameters [4]. An example of a modelled house is shown in Figure 1.

Multiple types of energy can flow in the house (e.g. gas, electricity, heat). These types of energy are modelled as streams transporting one type of energy. These energy-types are converted, buffered and consumed by devices. Furthermore, energy-types can be exchanged with the environment, which is modelled by exchanging devices. Every device can have certain energy-streams flowing in and certain energystreams flowing out, e.g. a microCHP has a gas stream in and an electricity and a heat stream out.

Energy flows between devices, i.e. the energy-streams of the devices are connected with each other. Sometimes the energy flows directly from one device to one other device (e.g. heat from the boiler to the central heating) while in other cases energy can flow from and to multiple devices (e.g. electricity). Therefore, pools are introduced. Energy-streams of the devices are connected to a pool. One or more energy-streams can flow into the same pool and one ore more energy-streams can flow out of the same pool.

Since a discrete simulation is used, the simulation horizon is discretisized resulting in a set of consecutive time intervals. Every time interval the pools in the house need to be in balance, i.e. as much energy must flow into the pool as flows 
out. A detailed description of the model and the simulator can be found in [4].

The balance in the pools can be reached, both in the simulation as in real-world scenarios, by using the flexibility of devices: some devices can vary the amount of energy flowing in and/or out. For example, a boiler can be switched on or off, the amount of electricity imported from the grid can vary, a certain amount of energy can be stored or supplied by a buffering device and some consuming devices can be shifted in time. The decisions influence the energy efficiency, electricity import profile, etc. and therefore some decisions are more desirable than others. The goal of the local controller is to make good decisions given a certain objective (e.g. peak shaving or following a global objective). The local controller can work independently or cooperating in the global three step methodology. The steering signals from the global controller are incorporated as energy import/export prices. When a local optimization is used, the objective is also incorporated using the energy import/export prices. The control algorithm used for this model is based on the control algorithm described in [7] and is the third step of the methodology.

This control methodology is implemented on a small embedded PC to verify whether it is possible to run the methodology on an energy-efficient device. After all, the additional hardware required for optimization should consume less energy than is saved. Preferably, the complete methodology should eventually be implemented on a device already available, for example the smart energy meter.

Two large scale use cases are simulated. The first use case concerns the behavior of a microCHP device in 200 houses and the second use case concerns charging 100 electrical cars. The goal of these simulations is on the one hand to verify different parameters of the optimization methodology and on the other hand to investigate how much potential can be exploited. The studied parameters of the optimization methodology are:

- level of optimization - global or local optimization, planning or realtime optimization

- individual steering signals or one shared steering signal

- the influence of prediction errors

The potential that should be exploited in the first use case is the ability to shift electricity production in time to reduce imbalance, for example caused by wind turbines. In the second use case the total required charge power should be flattened over the complete night instead of a large peak when everybody arrives at home at the end of the day.

\section{LOCAL NODE}

The goal of the three step methodology is to increase the overall energy efficiency. As a consequence, the introduction of a control system with distributed control should save more energy (or increase the efficiency) than requires for itself. Therefore, a proof of concept distributed control system is designed which can be placed in buildings. This node can communicate with all (controllable) appliances and the global controller. Furthermore, it runs the local control software. In other words, the node is responsible for the energy profile predictions, its corresponding optimization potential and the real time control.
Power consumption of the node, including the control hardware of the appliances, should be kept low. For our node, a E-Box 3300 using only $5 \mathrm{~W}$ is used in combination with the PlugWise measuring and switching hardware, using up to a $9 \mathrm{~W}$ in total. Although the system is low-power, it still has enough computational power to run our algorithms. Our predictions algorithms require between 4 up to roughly 7000 millisecond to run. Concretely, a complete planning process can be executed within a couple of minutes. This system consists of off-the-shelf components, so the power usage could be reduced even further.

Since this system is switched on continuously, the minimal saving of the system must be $79 \mathrm{kWh}$ per year. These savings can be obtained using different kind of techniques. First, user awareness of their energy consumption already leads to a reduction of energy usage. Due to the connectivity possibilities of the node, constant information about the electricity consumption can be displayed on a TV, LCD display or be accessed via the web/smart phones etc. Tests in the Netherlands show that energy aware households can save up to $200 \mathrm{kWh}$ [8]. Furthermore, since the electricity consumption is analyzed by the node, energy wasted by appliances in stand-by mode can be reduced by switching them off when people are not at home. A typical Dutch household consumes $400 \mathrm{kWh}$ on stand-by appliances, of which $50 \%$ can be saved by really switching of the appliances.

Besides the power savings, using the control box an improved energy profile can be shaped which can be supplied more efficiently, leading to a reduced $\mathrm{CO}_{2}$ emission while generating the required electricity. About half of a household's electricity demand is dedicated to controllable load (fridges, heaters, washing machines etc) [9], which could be scheduled (within certain limits). Thus although no electricity is saved, less $\mathrm{CO}_{2}$ may be required to generate the same amount of electricity if the household can be controlled.

Thus although adding a smart energy-management node will require some extra energy, more energy can be saved with the system. Furthermore, it allows a large part of the whole energy consumption to be generated more efficiently. On top of the environmental benefits, more comfort can be offered to the residents.

\section{Simulations}

The two simulated uses cases are large scale simulations. Large scale simulations are more realistic since prediction errors, peaks, etc. may level out in a large group. We chose for a group of 200 houses since this is the maximum number behind one lowest-level-transformer $(220 \mathrm{~V})$ in the Netherlands The 100 electrical cars is based on a penetration level of $50 \%$. Each use case has a certain goal, which we want to verify (e.g. the influence of prediction errors). Next, each use cases has a certain optimization objective, the optimization methodology works towards an objective (e.g. peak shaving). The use cases are simulated with different levels of optimization and with and without prediction errors. Since the simulations are discrete, the simulation horizon is divided in time intervals.

The electricity grid must always be in balance, i.e. as much generation as consumption. Therefore, all electricity produc- 
tion and consumption is planned or predicted on beforehand. The grid operator makes sure that production and consumption are equal at all times. A deviation from this plan/prediction causes an imbalance and penalties for the one causing the imbalance. For example, the amount of required charge power for the electrical cars is predicted and a power plant generates the predicted amount of electricity. A deviation from the predicted pattern must be solved by the power plant generating more or less electricity than planned. The microCHP devices in the houses are in first instance used to supply the heat demand. Therefore, the electricity production is limited by the heat demand and the size of the heat buffer (a part of the heat can be produced before it is consumed). Also these electricity production patterns are used in the balancing process of the grid operator so the microCHPs should generate electricity on the time they predicted.

The first use case concerns 200 houses with a microCHP device producing heat and electricity simultaneously. The optimization objective of the first use case is twofold. On the one hand, a group of houses is planned to produce a more or less stable (flat) electricity output for a complete day, which is to be followed by the realtime control mentioned before. Next to this, part of the scheduling freedom in the planning process is reserved for the opportunity to balance unpredictable mismatches in the electricity grid. In this use case individual steering signals are used (one per house) and only a global level of optimization since the imbalance is on a global level. The goal of the simulation is to investigate the ability to reduce the overall imbalance and the influence of prediction errors. The time interval length in this use case is six minutes and the simulation horizon 24 hours.

The second use case concerns charging 100 electrical cars when they arrive at home in the afternoon/evening. The optimization objective is to flatten the required charge power pattern. Without management all cars would start charging when they arrive at home. The goal is to compare different levels of optimization and to investigate the ability to flatten the overall charge pattern, also with prediction errors. One shared steering signal is used with the global optimization, an electricity import price. The time interval length is five minutes and the simulation horizon is 13 hours (5pm to 6am).

Comparing the results of our methodology with other methodologies is hard since different scenarios and use cases are used to verify them. Details of the tests are often not given so it is not possible to use the tests for another methodology. In [10] microCHPs are also used for compensating imbalance (caused by wind turbines). But all generation capacity is used as balancing power without taking into account that the total electricity generation is fixed and only can be shifted in time (see description below). Under these assumptions, a reduction of imbalance power up to $73 \%$ is reached.

\section{A. Balancing power by microCHP}

The first use case consists of a group of 200 houses, which is regarded as a local unit behind the lowest transformer level in the electricity grid. Since this group of houses is geographically located around the same place, we assume a high level of similarity between the characteristics of these houses. For this reason the microCHP and heat buffer that are used in each house have the same parameter settings. The heat demand in the houses differs per house; however, the total demand is similar (the maximum and minimum total heat demands are $63064 \mathrm{Wh}$ and $43544 \mathrm{Wh}$ respectively). In all cases the heat demand always has to be fulfilled, meaning that the limits cannot be violated.

The microCHP that is used produces an electricity output of $1 \mathrm{~kW}$, with a corresponding heat output of $8 \mathrm{~kW}$. It does not immediately produce these amounts; a startup period of 12 minutes is modelled, during which the production of heat and electricity linearly increase from 0 to their respective maximal values. After the microCHP is switched off, the production linearly decreases to 0 in a period of 6 minutes. The heat buffer capacity is $10 \mathrm{kWh}$; in the planning additional boundaries of minimally $500 \mathrm{Wh}$ and maximally $9500 \mathrm{Wh}$ are used to reserve balancing power. Initial buffer levels vary between 1 and $9 \mathrm{kWh}$, according to the following (in $\mathrm{kWh}$ ):

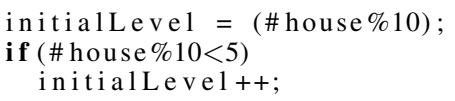

The heat demand is generated as in Algorithm 1 in [11], using $s=0, w=4$ and $I_{\text {season }}=I_{\text {winter }}$ for houses $0-99$ and $s=1, w=4$ and $I_{\text {season }}=I_{\text {winter }}$ for houses $100-199$, resulting in heat demand profiles with two peaks (one around 7-10 am and one around 6-9 pm).

As mentioned before, a more or less stable production planning is required for the group of houses. A lower bound is set to $32 \mathrm{~kW}$ and an upper bound to $82 \mathrm{~kW}$. These bounds are used to penalize under- and overproduction. A local search method based on the iterative use of a Dynamic Programming method for single houses [12] is used to find an hourly planning that minimizes the penalties incurred from exceeding these bounds. The goal for the realtime control is to follow the planning and utilize the scheduling freedom in this planning.

As explained above, imbalance is a deviation from the predicted consumption/generation pattern. Since the total electricity production of a microCHP is fixed (all heat demand must be supplied), the predicted production pattern of the houses should be known and published on beforehand. Next, an imbalance pattern is added, emulating imbalance caused by prediction errors in the production of wind turbines. This imbalance pattern is used to verify how much imbalance can be compensated. No extra or less electricity can be generated by the houses (heat demand defines the amount of generation), the generation can only be shifted in time. Therefore, the sum of the imbalance pattern is set to zero. The imbalance pattern is generated randomly between $+20 \mathrm{~kW}$ and $-20 \mathrm{~kW}$ and normalized so the sum is zero (as explained earlier). The eventual imbalance is defined as the deviation from the predicted generation pattern:

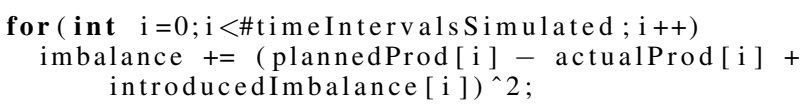

For the realtime global optimization all microCHPs send their status to the global controller. The global controller selects, based on the imbalance at the moment, a number of 
TABLE I

RESULTS USE CASE 1: TOTAL IMBALANCE (IN KW²)

\begin{tabular}{|c|c|c|c|}
\hline \multirow{3}{*}{$\begin{array}{c}\text { Prediction error } \\
\text { in heat consumption }\end{array}$} & $\begin{array}{c}\text { No imbalance } \\
\text { introduced }\end{array}$ & $\begin{array}{c}\text { Imbalance } \\
\text { no optimization }\end{array}$ & $\begin{array}{c}\text { Imbalance } \\
\text { optimization }\end{array}$ \\
\hline No & 1289 & 4075 & 2516 \\
Yes & 4004 & 5975 & 4833 \\
\hline
\end{tabular}

microCHPs to switch on/off. The individual steering signal of the selected microCHP devices are changed.

Six different scenarios are simulated:

- No imbalance and no prediction errors - determine the imbalance caused by simplifications in the models used for planning

- Imbalance, no imbalance compensation and no prediction errors - determine the initial imbalance

- Imbalance, imbalance compensation and no prediction errors - determine imbalance reduction potential

- No imbalance and prediction errors

- Imbalance, no imbalance compensation and prediction errors

- Imbalance, imbalance compensation and prediction errors

1) Results: All simulation results can be found in Table I, the planning and actual production of the first and third scenario are given in Figure 2. As can be seen in the table, the initial case (no imbalance) already has an imbalance of $1289 \mathrm{~kW}^{2}$. After adding the imbalance pattern (an extra $2689 \mathrm{~kW}^{2}$ ) the total imbalance is $4075 \mathrm{~kW}^{2}$. The imbalance pattern is given in Figure 2. In the third, optimizing scenario the imbalance is $2516 \mathrm{~kW}^{2}$, a reduction of $38 \%$ compared with the second scenario.

When a prediction error is introduced the initial imbalance increases significantly $\left(4004 \mathrm{~kW}^{2}\right)$. The local and global controller do not react on prediction errors, they try to reach the predicted production pattern anyhow (per individual microCHP device due to the individual steering signals). The imbalance pattern increases the imbalance to $5975 \mathrm{~kW}^{2}$, optimization decreases it to $4833 \mathrm{~kW}^{2}(19 \%)$.

\section{B. Charging electrical cars}

All cars have the same charge current $(1.5 \mathrm{~kW})$ but the required charging time differs between one and four hours (based on current available electrical cars). The charge time increases from one hour for the first car to four hours for the last car with a total charge time of 261 hours $(391 \mathrm{kWh})$ :

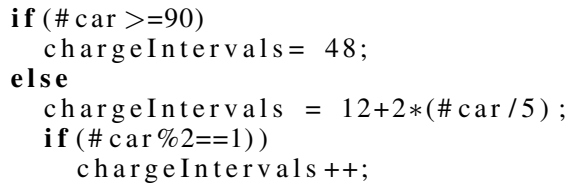

The cars arrive at home between $5 \mathrm{pm}$ and $8 \mathrm{pm}$ and they must be charged at $6 \mathrm{am}$ the next morning. The charge time depends on the number of the car, so the arrival time should be randomly distributed. To randomize the arrival times, the pseudo-random development of the coefficients of $\pi$ are used (so the use case can be reproduced):

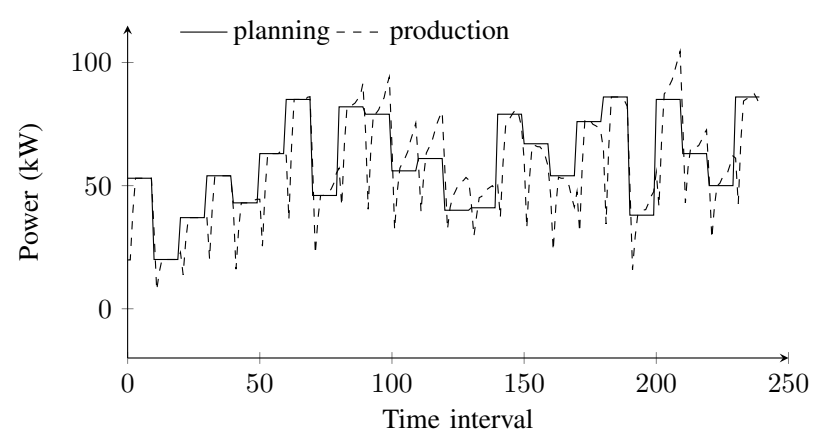

(a) scenario no imbalance

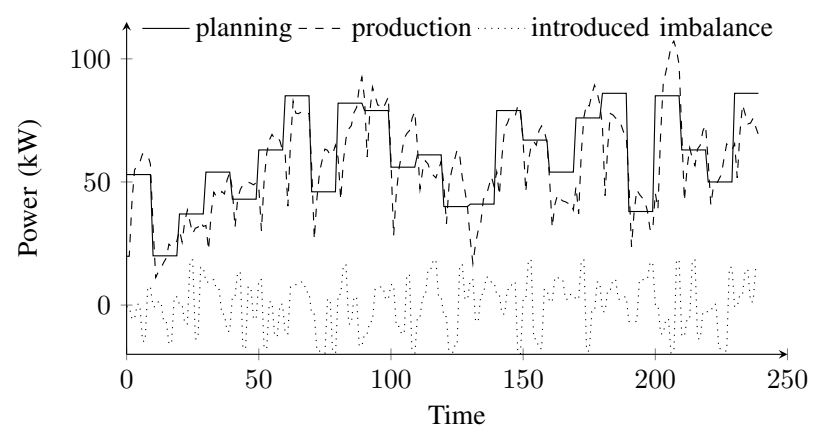

(b) scenario optimization

Fig. 2. Use case one: planning and resulting production patterns

arrivalTime $=204+\operatorname{piCoef}[\# \mathrm{car}] * 4$

With this information the use case (arrival time and charge time) can be generated. Since the goal is to reduce the peaks, the results are evaluated based on the average/peak ratio (load factor, higher is better) of the total charge current (the highest peak divided by the average) and the imbalance power. The imbalance power is in this case defined as the deviation from the average $(391 \mathrm{kWh} / 13$ hours $\approx 30 \mathrm{~kW}, 20$ cars charging):

for (int $\mathrm{i}=0 ; \mathrm{i}<\#$ timeIntervalsSimulated; $\mathrm{i}++$ )

imbalance $+=(\text { ElectricityDelivered }[\mathrm{i}]-30.000)^{\wedge} 2$;

This use case is simulated with four different levels of optimization. The simulated optimization levels are:

- no optimization - cars start charging when they arrive at home,

- local realtime optimization - based on the status (required charge time and time left until 6am) the carchargers individually decide when they charge,

- global realtime optimization - every time interval all cars communicate their status to a global controller, this global controller distributes a steering signal based on the status and a prediction of the total required charge power,

- planning - using the iterative approach and predictions of the arrival and charge time a near-optimal planning is deducted (based on one shared steering signal per time interval). Both perfect predictions and prediction errors are simulated.

1) Results: The results of the simulations are shown in Figure 3 and Table II and discussed in more details in the following paragraphs. When no optimization is used, all cars 
TABLE II

RESULTS USE CASE 2

\begin{tabular}{|c|c|c|c|c|c|c|c|c|}
\hline & \multicolumn{2}{|c|}{ 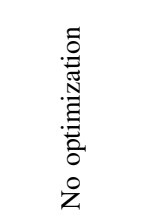 } & \multicolumn{2}{|c|}{ 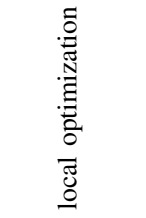 } & \multicolumn{2}{|c|}{ 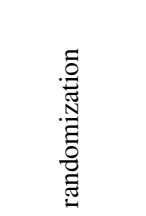 } & \multicolumn{2}{|c|}{ 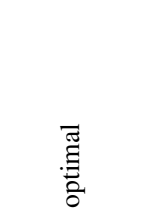 } \\
\hline $\begin{array}{l}\text { imbalance } \\
\left(\mathrm{kW}^{2} \cdot 10^{3}\right) \\
\text { load factor }\end{array}$ & \multicolumn{2}{|c|}{223} & \multicolumn{2}{|c|}{364} & \multicolumn{2}{|c|}{29} & \multicolumn{2}{|c|}{2} \\
\hline & \multicolumn{8}{|c|}{$\begin{array}{c}\text { Global realtime optimization } \\
\text { (predicted average number of cars charging) }\end{array}$} \\
\hline & 15 & 16 & 17 & 18 & 19 & 20 & 21 & 22 \\
\hline $\begin{array}{l}\text { imbalance } \\
\left(\mathrm{kW}^{2} \cdot 10^{3}\right)\end{array}$ & 12 & 10 & 7 & 7 & 7 & 11 & 12 & 19 \\
\hline load factor & 0.59 & 0.59 & 0.61 & 0.61 & 0.65 & 0.65 & 0.65 & 0.63 \\
\hline
\end{tabular}

start charging the moment they arrive at home. This results in a peak in the begin.

When a local controller is used, no steering signals from the global controller are incorporated in the decision. The decision of the local controller whether to shift charging in time or not, is based on the status of the charger (required charge time left and the total time left). This results in an inferior charge pattern (see Figure 3(b)). The states converge for all chargers since they all use the same decision parameters resulting in very high peaks when all cars decide to charge at the same moment. However, when a random factor is added to the cost function the results are much better. Due to the large number of cars the randomization results in an uniform distribution (since the random function is uniform distributed).

The global realtime control algorithm determines every time interval a steering signal. Based on a prediction of the total required charge power the average charge power per interval is determined (one time, at the begin of the optimization period). Every time interval, all local controllers send their status to the global controller. Based on this information and the predicted average charge power the steering signal can be determined, i.e. determine a signal so that 20 cars will charge. The status of all chargers are ordered and then the steering signal is adjusted to the $20^{t h}$ position in the list. Using this approach, at least 20 cars will charge since the status of at least 20 cars are such that they react. However, more than 20 cars can react on the steering signal, e.g. when the status of position 21 in the ordered list is equal to the status of position 20. Furthermore, the prediction of the total required charging power can be wrong. Therefore, a predicted number of cars charging between 15 and 22 are simulated. The results of this simulations are given in Table II. As can be seen, a too low prediction of the predicted charge power results in a better performance than a too high prediction (due to the fact that more than the desired number of charger can react on the steering signal).

The iterative planning approach has two important parameters: the number of iterations and how much the steering are adjusted per iteration. To find an optimal schedule, first

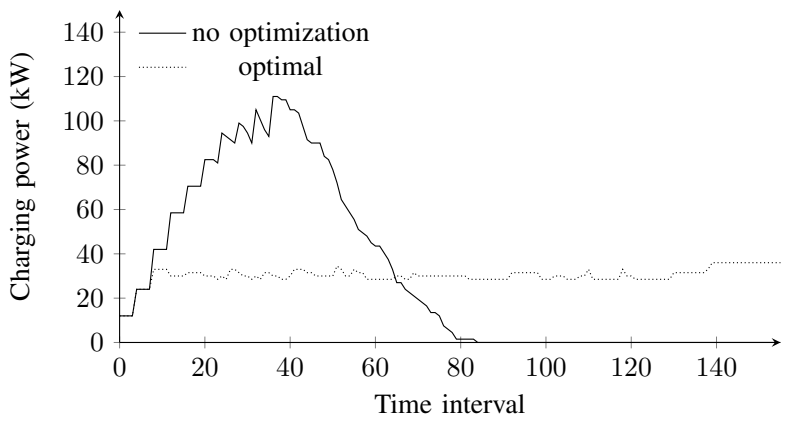

(a) Scenario without optimization and the optimal charge schedule

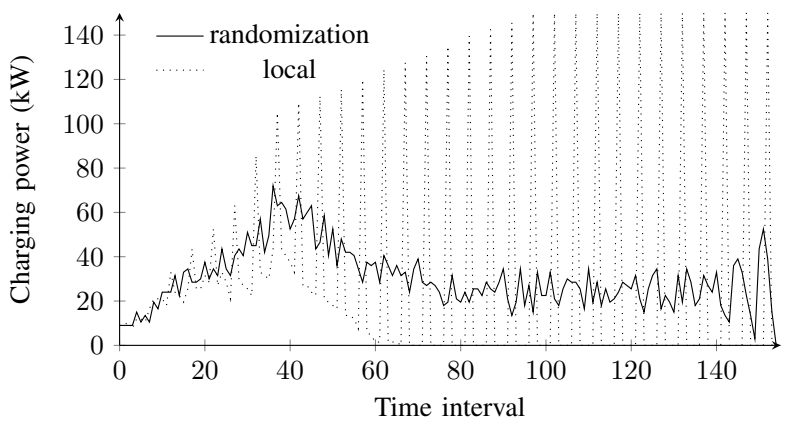

(b) Scenario local optimization and randomization

Fig. 3. Use case 2: total charge power

the number of iterations is set to 80 and the steering signal adjustment to 1 . This results in an optimum schedule (using one steering signal) with an imbalance power of $2000 \mathrm{~kW}^{2}$. However, 80 iterations are not realistic due to the exhaustive communication this requires. A reasonable tradeoff between quality of the schedule and communication costs is 20 iterations and an adjustment of 2, resulting in an imbalance of $4000 \mathrm{~kW}^{2}$. On top of this schedule, a prediction error is introduced. The number of charge intervals is calculated using the above given function, this number is used as prediction and during simulation a variation is added to this number. For this variation the pseudo-random development of the coefficients of $\pi$ is again used:

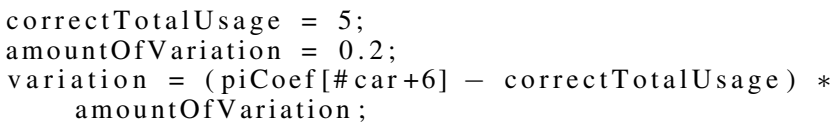

The first 6 coefficients are not used to prevent a relation between starting time and variation ( 6 is arbitrary chosen, multiple values are simulated, all with similar results). The parameter correctTotalUsage defines whether the total charge power is equal (only variation), lower or higher. Three different values for the parameter are simulated: 5 (equal usage), 2 (more charge power) and 8 (less charge power). The parameter amount $\mathrm{f}$ Variation defines the amount of variation. The results of these simulation are given in Figure 4. When only variation is added while the total charge power is equal, the planning can be followed quite well and only a little extra imbalance is introduced. The large number of houses levels out the variation, due to the uniform distributed distribution. The shared steering signal and the same cost function for every 


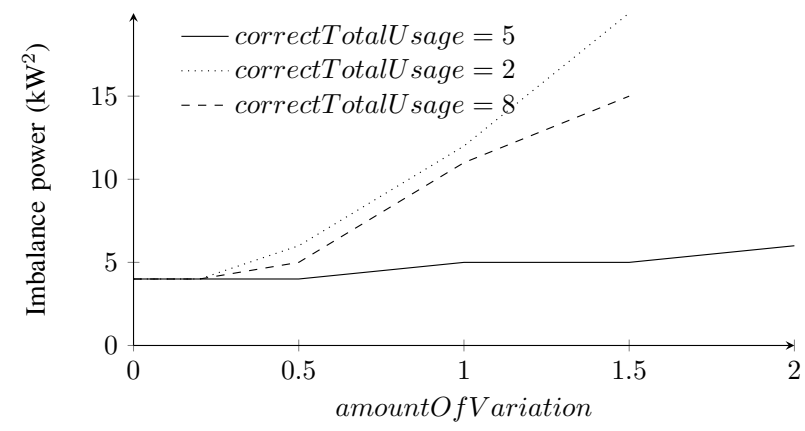

Fig. 4. Resulting charge power planning and prediction errors

chargers result in houses with extra charge time react when houses with less charge power does not react. When the total charge power also deviates, the imbalance power increases significantly. Just as with the realtime global control, when more charge power is required than predicted the errors are larger than when less charge power is required.

\section{DiscusSiON}

The implementation of the proof-of-concept local controller on a embedded PC shows that it is possible to run the developed algorithms on such rather simple systems. The energy usage of such a system can already be compensated by the electricity usage reduction caused by the awareness of residents. More energy reduction can be reached due to increasing efficiency of power plants and enabling the introduction of renewable sources, but how much reduction is very hard to predict and calculate.

The first use case shows that it is possible to decrease the imbalance up to almost $40 \%$ by only shifting the predicted electricity generation in time. Generators with a complete freedom of runtime (e.g. a diesel generator) or a battery can probably decrease the imbalance even more. The second use case shows that it is possible to flatten the consumption pattern of a large group of electrical cars, the load factor increased from 0.27 to 0.84 ( 1 is the maximum, higher is better). Local optimization gives very bad results, due to similarities between the chargers and situations the optimization functions converge resulting in very high peaks. Adding randomization to this local optimization increases the results significantly (better than no optimization), but this is unpredictable and uncontrollable. A realtime global control or a global planning gives the best results but require much more communication.

One steering signal seems to perform better when prediction errors are introduced. The large number of houses levels out the prediction errors: a house with a higher usage than predicted reacts instead of a house with a lower usage than predicted. On the contrary, when individual steering signals are used every house tries to reach its individual predicted pattern resulting in much higher deviations from the pattern. In this case a realtime algorithm to compensate for prediction errors is required, preferable on a global level so houses can compensate for each other. However, with individual steering signals a more detailed pattern can be reached since multiple houses can react on one shared signal, as can be seen on the imbalance in the car charge scenario without prediction errors.

Furthermore, a realtime global controller can react better on prediction errors than a planning. However, it requires much more communication and it can only be used in simple cases (e.g. charging batteries) since no (predicted) information of individual houses is available, which is required for e.g. the microCHP planning. The best solution seems to be a combination of planning and global realtime optimization, but this requires a lot of communication. Probably the best solution is planning and re-planning when too much deviation from the planning is detected, using new, short-term predictions. This is topic of current research.

Summarizing, it is possible to implement a local controller that consumes less energy than it saves. Furthermore, simulations show that it is possible to exploit potential and optimize behavior. A global level of optimization is required and it seems better to use one shared steering signal since prediction errors level out for a large group.

\section{ACKNOWLEDGEMENT}

This research is conducted within the Islanded House project supported by E.ON Engineering and the SFEER project supported by Essent, Gasterra and STW.

\section{REFERENCES}

[1] A. Molderink, V. Bakker, M. Bosman, J. Hurink, and G. Smit, "A threestep methodology to improve domestic energy efficiency," in IEEE PES Conference on Innovative Smart Grid Technologies, 2010.

[2] A. de Jong, E.-J. Bakker, J. Dam, and H. van Wolferen, "Technisch energie- en CO2-besparingspotentieel in Nederland (2010-2030),' Platform Nieuw Gas, p. 45, Juli 2006.

[3] A. Molderink, M. Bosman, V. Bakker, J. Hurink, and G. Smit, "Hardand software implementation and verification of an islanded house prototype," in International Conference on System Engineering. IEEE, 2009.

[4] V. Bakker, A. Molderink, M. G. C. Bosman, J. L. Hurink, and G. J. M. Smit, "On simulating the effect on the energy efficiency of smart grid technologies," in Proceedings of the 2010 Winter Simulation Conference submitted, 2010.

[5] V. Bakker, M. G. C. Bosman, A. Molderink, J. L. Hurink, and G. J. M. Smit, "Demand side load management using a three step optimization methodology," in Proceedings of the 2010 IEEE Conference Smart Grid Communication accepted, 2010.

[6] A. Molderink, V. Bakker, M. G. C. Bosman, J. L. Hurink, and G. J. M. Smit, "Management and control of domestic smart grid technology," IEEE Transactions on Smart Grid, vol. 1, no. 2, 2010.

[7] A. Molderink, V. Bakker, M. Bosman, J. Hurink, and G. Smit, "Domestic energy management methodology for optimizing efficiency in smart grids," in IEEE conference on Power Technology. IEEE, 2009.

[8] http://www.milieucentraal.nl/pagina?onderwerp=Apparaten.

[9] C. Block, D. Neumann, and C. Weinhardt, "A market mechanism for energy allocation in micro-chp grids," in 41st Hawaii International Conference on System Sciences, Jan 2008, pp. 172-180.

[10] M. Houwing, P. G., P. W. Heijnen, and I. M.D., "Balancing wind power with virtual power plants of micro-chps," in Proceedings of 2009 IEEE Power Tech Conference. IEEE, 2009.

[11] M. Bosman, V. Bakker, A. Molderink, J. Hurink, and G. Smit, "Benchmarking set for domestic smart grid management (submitted),' June 2010.

[12] - "Production planning in a virtual power plant," in Proceedings of the 20th annual workshop on Program for Research on Integrated Systems and Circuits, Veldhoven, Netherlands. Utrecht: Technology Foundation STW, November 2009, p. 6. 